\title{
Two-Level Atom at Finite Temperature
}

\author{
T. SOWIŃSKI* \\ Center for Theoretical Physics, Polish Academy of Sciences, al. Lotników 32/46, 02-668 Warsaw, Poland \\ and \\ Faculty of Biology and Environmental Sciences, Cardinal Stefan Wyszynski University \\ Wóycickiego 1/3, 01-938 Warsaw, Poland
}

(Received October 26, 2009)

\begin{abstract}
Properties of a two-level atom coupled to the quantized electromagnetic field at finite temperature are determined. The analysis is based on a new method (inspired by quantum electrodynamics) of describing qubits, developed previously at zero temperature. In this paper, we make a generalization to finite temperature by introducing the Matsubara formalism and the temperature propagators. We analyze the spectral properties of different types of propagators and we derive a direct connection between the temperature propagators and the real time propagators. To show the effectiveness of this method, we calculate the temperature dependence of the polarizability of a two-level atom in the lowest order of perturbation theory and we predict an unexpected sharpening of the resonance. The whole discussion is carried out without the rotating wave approximation.
\end{abstract}

PACS numbers: 11.10.Wx, 32.10.Dk, 31.15.ap, 32.70.Jz

\section{Introduction}

The main purpose of this paper is to show that a new method of describing qubits within the quantum field theory formalism developed by us in [1] can be also used to describe qubits at finite temperature. The main idea comes from the observation made long time ago by Matsubara [2] that there exists a close analogy between the Feynman propagators and the so-called temperature propagators. In his paper, Matsubara described general rules of perturbation techniques at non-zero temperature based on the Feynman diagrams and used them with great success to describe the system made of electrons and positrons. The Matsubara formalism was further developed by many authors [3-6] and it has quickly become one of the main tools of modern statistical mechanics [7-10]. In the usual treatment the methods of quantum field theory are exploited to describe systems in which the number of particles is not conserved, i.e. in the grand canonical assemble. In this paper we will adopt this formalism to analyze the temperature properties of one qubit realized by precisely one electron in atomic states. It is not obvious that it is indeed possible. Therefore, at the beginning we present a short introduction to thermal field theory and we give a clear prescription how to proceed.

The influence of thermal fluctuations on the properties of qubits was partially discussed before. First, a very general treatment was based on dissipation phenomena in the damped cavity model [11-13]. The problem of the influence of the temperature on the induced inter-

\footnotetext{
* e-mail: tomsow@cft.edu.pl
}

actions between qubits via the electromagnetic field and on their entanglement is recently considered in the context of the so-called sudden death of entanglement phenomenon [14-16]. Very often this analysis is carried out in the Jaynes-Cummings model [17] in the rotating wave approximation (RWA) [18-21]. There exist also some numerical predictions on the behavior of qubits at finite temperatures realized in quantum dots [22]. So far a general problem of the dynamical properties of a single qubit interacting with the quantized electromagnetic field (without RWA) at finite temperature has not been discussed since all current techniques did not give such possibilities. In this paper we want to show that methods ingrained in quantum field theory could be the first step to solve this problem.

To make our whole argumentation clear, we restrict ourselves to the problem of a two-level atom and its polarizability (response tensor), since a two-level atom is the simplest model of the interaction between light and matter where purely mathematical problems are as small as possible [1]. Moreover, the properties of the linear polarizability of a two-level atom were discussed in detail at zero-temperature regime by many authors and many discussions on it took place [23-30]. These discussions have never gone beyond zero temperature case, since it seemed to be a very hard task.

The description of qubits interacting with quantized electromagnetic field introduced in [1] was used to resolve the old sign controversy but its usefulness is not limited to these problems. In this paper we want to show that our new method of describing qubits in terms of field operators is also a key to deal with finite temperature problems. As in the previous case, we will not apply RWA at any point of the analysis [28]. 


\section{Physical situation}

\subsection{The Hamiltonian of the system}

The starting point of our analysis is the well known Hamiltonian of the two-level atom interacting with the quantized electromagnetic field

$$
\widehat{\mathcal{H}}=\widehat{\mathcal{H}}_{0}+\widehat{\mathcal{H}}_{\mathrm{I}},
$$

where

$$
\begin{aligned}
& \widehat{\mathcal{H}}_{0}=m \sigma_{z}+\int_{0}^{\infty} \mathrm{d} k k a^{\dagger}(k) a(k), \\
& \widehat{\mathcal{H}}_{\mathrm{I}}=\sigma_{x} \int_{0}^{\infty} \mathrm{d} k g(k) \Phi(k) .
\end{aligned}
$$

The Hamiltonian $\widehat{\mathcal{H}}_{0}$ describes the properties of noninteracting subsystems - two-level atom with the energy gap equal to $2 m$ and the free quantized electromagnetic field. The operators $a^{\dagger}(k)$ and $a(k)$ create and annihilate photons in the appropriate modes of the electromagnetic field and together they form a quantum scalar field

$$
\Phi(k)=\frac{a(k)+a^{\dagger}(k)}{\sqrt{2 k}} .
$$

The Hamiltonian $\widehat{\mathcal{H}}_{\mathrm{I}}$ is responsible for the interaction between the photons and the two-level atom. The form factor $g(k)$ is simply a coupling coefficient measuring the strength of a dipole transition in the atom. Let us note that we do not use a rotating wave approximation in the coupling Hamiltonian (2b). As was shown previously in [28], the complete interaction term is necessary to obtain a correct expression for the polarizability of a two-level atom.

\subsection{Two-level atom in thermal equilibrium}

In our treatment of a qubit at non-zero temperature we will make two physical assumptions. First that our system described by the Hamiltonian (1) is in thermal equilibrium with the heat reservoir (thermostat) at temperature $T$. Second that the temperature is not so high as to induce the creation of real electron-positron pairs. It means that the number of fermions in our system is conserved. Notice however that we do not assume that the number of photons in the system is conserved.

Description of the statistical properties of a quantum system in thermodynamic equilibrium is realized in terms of the statistical assemblies. In our model an appropriate assembly is defined by the following control parameters:

- the number of fermions (electrons) equal to 1 ,

- the temperature $T$ of the thermostat,

- the chemical potential $\mu$ of the photon reservoir.

It is worth to notice that because of the properties of the electromagnetic field, the chemical potential $\mu$ is always equal to 0 . It means that all statistical properties of the photons can be expressed as functions of the temperature $T$ and one extensive parameter (for example the volume of the cavity). Nevertheless, to emphasize the fact that $\mu$ is one of our control parameter, we will write it explicitly to the end of this section.

\subsection{Statistical operator}

From the rules of quantum statistical physics (cf., for example [31]) we know that any system in equilibrium with the thermostat and the reservoir of particles is in the quantum state represented by the following density matrix (in this context also called statistical operator)

$$
\widehat{\rho}=\frac{1}{\widehat{\mathcal{Z}}} \mathrm{e}^{-\beta \widehat{\mathcal{K}}}, \quad \widehat{\mathcal{Z}}=\operatorname{Tr}\left(\mathrm{e}^{-\beta \widehat{\mathcal{K}}}\right) .
$$

As always $\beta=1 / k T$ and $\widehat{\mathcal{Z}}$ is partition function. In our case the statistical Hamiltonian $\widehat{\mathcal{K}}$ is given by

$$
\widehat{\mathcal{K}}=\widehat{\mathcal{H}}-\mu \mathcal{N},
$$

where the operator $\mathcal{N}$ represents the number of photons in the system. Expectation value of any operator $\mathcal{O}$ in such a quantum state of the system is given by

$$
\langle\langle\mathcal{O}\rangle\rangle=\operatorname{Tr}(\widehat{\rho} \mathcal{O})=\frac{\operatorname{Tr}\left(\mathrm{e}^{-\beta \widehat{\mathcal{K}}} \mathcal{O}\right)}{\operatorname{Tr}\left(\mathrm{e}^{-\beta \widehat{\mathcal{K}}}\right)} .
$$

For future needs let us now introduce an additional system composed of a non-interacting two-level atom and the electromagnetic field. We define the free statistical Hamiltonian $\widehat{\mathcal{K}}_{0}$ and the free statistical operator $\widehat{\rho}_{0}$ of such a system as follows:

$$
\begin{aligned}
& \widehat{\mathcal{K}}_{0}=\widehat{\mathcal{H}}_{0}-\mu \mathcal{N}, \\
& \widehat{\rho}_{0}=\frac{1}{\widehat{\mathcal{Z}}_{0}} \mathrm{e}^{-\beta \widehat{\mathcal{K}}_{0}}, \quad \widehat{\mathcal{Z}}_{0}=\operatorname{Tr}\left(\mathrm{e}^{-\beta \widehat{\mathcal{K}}_{0}}\right) .
\end{aligned}
$$

The statistical operator $\widehat{\rho}_{0}$ describes a quantum state of a system composed of a free two-level atom and the free electromagnetic field which together are in equilibrium with the thermostat and the reservoir of photons. The connection between statistical Hamiltonians in the interacting and the non-interacting case is the following:

$$
\widehat{\mathcal{K}}=\widehat{\mathcal{K}}_{0}+\widehat{\mathcal{H}}_{\mathrm{I}}
$$

and the expectation value of the operator $\mathcal{O}$ in the state $\widehat{\rho}_{0}$ is given by

$$
\langle\langle\mathcal{O}\rangle\rangle_{0}=\operatorname{Tr}\left(\widehat{\rho}_{0} \mathcal{O}\right)=\frac{\operatorname{Tr}\left(\mathrm{e}^{-\beta \widehat{\mathcal{K}}_{0}} \mathcal{O}\right)}{\operatorname{Tr}\left(\mathrm{e}^{-\beta \widehat{\mathcal{K}}_{0}}\right)} .
$$

\subsection{Linear polarizability of the atom}

The linear polarizability of a two-level atom is a physical measure of the reaction of the system to small external electromagnetic perturbations. When the state of the system is described by the statistical operator (4a), then the polarizability is defined as the following two-point retarded correlation function $[32,33]$ :

$$
\begin{aligned}
& \mathfrak{a}\left(t, t^{\prime}\right)=-\mathrm{i} A \theta\left(t-t^{\prime}\right) \\
& \quad \times\left\langle\left\langle\left[\mathrm{e}^{\mathrm{i} \hat{\mathcal{H}} t} \sigma_{x} \mathrm{e}^{-\mathrm{i} \widehat{\mathcal{H}} t}, \mathrm{e}^{\mathrm{i} \widehat{\mathcal{H}} t^{\prime}} \sigma_{x} \mathrm{e}^{-\mathrm{i} \widehat{\mathcal{H}} t^{\prime}}\right]\right\rangle\right\rangle .
\end{aligned}
$$

For a single two-level atom, the constant $A$ is equal to $d^{2} / 2 \hbar$, where $d$ is a dipole-moment of the atomic transition. It is easy to find that $\mathfrak{a}\left(t, t^{\prime}\right)$ depends only on the difference of its arguments and therefore it has a very simple form in the frequency domain 


$$
\mathfrak{a}(\omega)=\int_{-\infty}^{\infty} \mathrm{d} t \mathrm{e}^{\mathrm{i} \omega\left(t-t^{\prime}\right)} \mathfrak{a}\left(t, t^{\prime}\right) .
$$

In this paper we give a precise and clear prescription how to find this quantity as a perturbation series using the well known methods of thermal quantum field theory $[2,7-10]$.

\section{Second quantization}

The basic ingredients of quantum field theory are obviously the field operators. Therefore, before we start to analyze our system in this language, we have to introduce, besides the electromagnetic field operator (3), the fermionic field operator representing the states of an electron in the atom. As usual, field operators can be introduced after performing second quantization of electronic states. In this model this procedure is very easy. We just enlarge the two-dimensional Hilbert space spanned by vectors $|g\rangle$ and $|e\rangle$ representing ground state and excited state of the electron in the atom to the four-dimensional one spanned by four state vectors: two previously defined $|\mathrm{g}\rangle$ and $|\mathrm{e}\rangle$, the zero-electron state $|\mathrm{N}\rangle$, and the antisymmetric two-electron state $|\mathrm{B}\rangle$ representing two electrons - one in each state.

Such a procedure enables one to introduce creation and annihilation operators of the electron. The operators $\psi_{\downarrow}$ and $\psi_{\downarrow}^{\dagger}$ annihilate and create electron in the ground state of the two-level atom, and $\psi_{\uparrow}$ and $\psi_{\uparrow}^{\dagger}$ do so in the excited state. These operators form together the fermion field operator and its Hermitian conjugate [1]

$$
\Psi=\left(\begin{array}{c}
\psi_{\uparrow} \\
\psi_{\downarrow}
\end{array}\right), \quad \Psi^{\dagger}=\left(\psi_{\uparrow}^{\dagger}, \psi_{\downarrow}^{\dagger}\right) .
$$

Then one can easily find the second-quantized form of the new Hamiltonian acting in the extended Hilbert space

$$
\mathcal{H}=\mathcal{H}_{0}+\mathcal{H}_{\mathrm{I}},
$$

where

$$
\begin{aligned}
& \mathcal{H}_{0}=m \Psi^{\dagger} \sigma_{z} \Psi+\int_{0}^{\infty} \mathrm{d} k k a^{\dagger}(k) a(k), \\
& \mathcal{H}_{\mathrm{I}}=\Psi^{\dagger} \sigma_{x} \Psi \int_{0}^{\infty} \mathrm{d} k g(k) \Phi(k) .
\end{aligned}
$$

The origin, and the properties of the Hamiltonian (12) and of the field operators $\Psi$ and $\Psi^{\dagger}$ are discussed in detail in our previous paper [1]. Notice that the system described by the Hamiltonian $\mathcal{H}$ may have states with different number of fermions. However, the number of fermions operator commutes with $\mathcal{H}$.

In the Heisenberg picture, the time evolution of the system is contained in the dynamics of the field operators. They evolve according to the following equations [1]:

$$
\begin{aligned}
& \left(\mathrm{i} \partial_{t}-m \sigma_{z}\right) \Psi(t)=\int_{0}^{\infty} \mathrm{d} k g(k) \Phi(k, t) \sigma_{x} \Psi(t), \\
& \left(\partial_{t}^{2}+k^{2}\right) \Phi(k, t)=-g(k) \Psi^{\dagger}(t) \sigma_{x} \Psi(t) .
\end{aligned}
$$

Now we introduce a new statistical operator $\rho$ representing the thermal equilibrium of the new quantum mechanical system

$$
\rho=\frac{1}{\mathcal{Z}} \mathrm{e}^{-\beta \mathcal{K}}, \quad \mathcal{Z}=\operatorname{Tr}\left(\mathrm{e}^{-\beta \mathcal{K}}\right),
$$

where the statistical Hamiltonian $\mathcal{K}$ is given by

$$
\mathcal{K}=\mathcal{H}-\mu \mathcal{N} .
$$

The expectation value of any operator $\mathcal{O}$ in the quantum state $\rho$ is given by

$$
\langle\mathcal{O}\rangle=\operatorname{Tr}(\rho \mathcal{O})=\frac{\operatorname{Tr}\left(\mathrm{e}^{-\beta \mathcal{K}} \mathcal{O}\right)}{\operatorname{Tr}\left(\mathrm{e}^{-\beta \mathcal{K}}\right)} .
$$

Notice that the expectation values (5) and (15), even for the same physical observable $\mathcal{O}$, are different since the statistical Hamiltonians $\mathcal{K}$ and $\widehat{\mathcal{K}}$ act in different Hilbert spaces. Nevertheless, for those qubit operators $\mathcal{O}_{1}, \ldots, \mathcal{O}_{n}$ that are represented by traceless $2 \times 2$ matrices (for example the free qubit Hamiltonian $m_{0} \sigma_{z}$, the interaction Hamiltonian (2b), or any other linear combination of the Pauli matrices) one can verify that there exists the following direct connection between the expectation value of their product $\left\langle\left\langle\mathcal{O}_{1} \ldots \mathcal{O}_{n}\right\rangle\right\rangle$ and expectation value of their second-quantized counterparts $\left\langle\Psi^{\dagger} \mathcal{O}_{1} \Psi \ldots \Psi^{\dagger} \mathcal{O}_{n} \Psi\right\rangle$ :

$$
\left\langle\left\langle\mathcal{O}_{1} \ldots \mathcal{O}_{n}\right\rangle\right\rangle=\frac{\mathcal{Z}}{\widehat{\mathcal{Z}}}\left\langle\Psi^{\dagger} \mathcal{O}_{1} \Psi \ldots \Psi^{\dagger} \mathcal{O}_{n} \Psi\right\rangle .
$$

It should be emphasized that this relation is a central theorem of this paper. It expresses nontrivial but a very simple connection between the expectation values of physical quantities of the system containing exactly one fermion and the expectation values of their non-physical second-quantized counterpart. Therefore, it is a peculiar link between the interesting physical system described by the Hamiltonian (1) and a convenient mathematical model described by the Hamiltonian (12). Without this connection further analysis would not be possible.

Relation (16) follows from the observation that for traceless matrices the bilinear combination of field operators $\Psi^{\dagger} \mathcal{O}_{i} \Psi$ gives zero when acting on the two-electron or the zero-electron states. Moreover, in the qubit (one-electron) subspace it acts as the qubit operator $\mathcal{O}_{i}$. Obviously, this argument is also valid for evolving qubit operators $\mathcal{O}_{i}(t)=\mathrm{e}^{\mathrm{i} \hat{\mathcal{H}} t} \mathcal{O}_{i} \mathrm{e}^{-\mathrm{i} \hat{\mathcal{H}} t}$ and their counterparts $\Psi^{\dagger}(t) \mathcal{O}_{i} \Psi(t)$.

This all means that a linear polarizability of the two-level atom defined by traceless $\sigma_{x}$ matrices in (9) can be reproduced from its second-quantized counterpart

$$
\begin{aligned}
& \alpha\left(t, t^{\prime}\right)=-\mathrm{i} A \theta\left(t-t^{\prime}\right) \\
& \quad \times\left\langle\left[\Psi^{\dagger}(t) \sigma_{x} \Psi(t), \Psi^{\dagger}\left(t^{\prime}\right) \sigma_{x} \Psi\left(t^{\prime}\right)\right]\right\rangle
\end{aligned}
$$

by using the property (16).

Let us note that function $\alpha\left(t, t^{\prime}\right)$ has no direct physical sense, since the statistical operator $\rho$ involves also non-physical states. Therefore we stress that we treat $\alpha\left(t, t^{\prime}\right)$ only as a very convenient tool to find a physical quantity.

\section{Matsubara formalism}

The starting point of the formulation of quantum field theory at finite temperatures is to introduce the temperature Green functions (or temperature correlation func- 
tions). They are defined in an analogous way as the Feynman Green functions at zero temperature. The difference lies in the evolution parameter - instead of the ordinary time variable $t$, we use the imaginary time $\tau=\mathrm{i} t$. As was first noticed by Matsubara in 1955 [2] after this simple replacing one can use almost all known machinery of the quantum field theory. In analogous to the ordinary time case all analysis is carried out in two quantum mechanical pictures - Heisenberg-like picture and Dirac-like picture.

In the Matsubara-Heisenberg picture the whole evolution of the system is contained in the evolution of the operators. Any operator $\mathcal{O}$ evolves according to the following rule:

$$
\mathcal{O}(\tau)=\mathrm{e}^{\mathcal{K} \tau} \mathcal{O} \mathrm{e}^{-\mathcal{K} \tau}
$$

In particular, the field operators (3) and (11) evolve in the following way:

$$
\begin{aligned}
& \Phi(k, \tau)=\mathrm{e}^{\mathcal{K} \tau} \Phi(k) \mathrm{e}^{-\mathcal{K} \tau}, \\
& \Psi(\tau)=\mathrm{e}^{\mathcal{K} \tau} \Psi \mathrm{e}^{-\mathcal{K} \tau}, \\
& \Psi^{\dagger}(\tau)=\mathrm{e}^{\mathcal{K} \tau} \Psi^{\dagger} \mathrm{e}^{-\mathcal{K} \tau} .
\end{aligned}
$$

In contrast, in the Matsubara-Dirac picture the operators evolve according to the free statistical Hamilto$\operatorname{nian} \mathcal{K}_{0}$ :

$$
O(\tau)=\mathrm{e}^{\mathcal{K}_{0} \tau} \mathcal{O} \mathrm{e}^{-\mathcal{K}_{0} \tau} .
$$

One can easily check that the field operators (3) and (11) evolve in the following way (from now we will put $\mu=0$ everywhere)

$$
\begin{aligned}
& \psi(\tau)=\left(\begin{array}{c}
\psi_{\uparrow} \mathrm{e}^{-m \tau} \\
\psi_{\downarrow} \mathrm{e}^{m \tau}
\end{array}\right), \\
& \psi^{\dagger}(\tau)=\left(\psi_{\uparrow}^{\dagger} \mathrm{e}^{m \tau}, \psi_{\downarrow}^{\dagger} \mathrm{e}^{-m \tau}\right), \\
& \phi(k, \tau)=\frac{a(k) \mathrm{e}^{-k \tau}+a^{\dagger}(k) \mathrm{e}^{k \tau}}{\sqrt{2 k}} .
\end{aligned}
$$

The interaction Hamiltonian (12c) in this picture has the form

$$
\begin{aligned}
& \mathcal{H}_{\mathrm{I}}(\tau)=\mathrm{e}^{\mathcal{K}_{0} \tau} \mathcal{H}_{\mathrm{I}} \mathrm{e}^{-\mathcal{K}_{0} \tau} \\
& =\psi^{\dagger}(\tau) \sigma_{x} \psi(\tau) \int_{0}^{\infty} \mathrm{d} k g(k) \phi(k, \tau) .
\end{aligned}
$$

\subsection{Temperature correlation functions}

Temperature correlation functions are the central objects of the quantum field theory at finite temperatures. In analogy to the Feynman ones, they do not have any direct physical interpretation, but they are the basic ingredients of the perturbation computations based on the Feynman diagrams.

We call the $n$-point temperature correlation function a complex function of $n$ parameters defined as follows (To underline the difference between imaginary and ordinary time functions we use a tilde.):

$$
\widetilde{\mathcal{G}}\left(\tau_{1}, \ldots, \tau_{n}\right)=-\left\langle\mathbb{T}_{\tau} \mathcal{O}\left(\tau_{1}\right) \cdots \mathcal{O}\left(\tau_{n}\right)\right\rangle,
$$

where the operator $\mathbb{T}_{\tau}$ is the time-ordering operator. This operator respects the fermionic nature of operators, and therefore for any odd permutation of electron operators one should remember about changing the sign of the expression. It will be clarified later that in practice it is sufficient to restrict each imaginary-time variable $\tau_{\mathrm{i}}$ only to the range

$$
0 \leq \tau_{\mathrm{i}} \leq \beta \text {. }
$$

Special case of temperature correlation functions (23) are temperature propagators of electromagnetic and fermion fields. They are defined as follows:

$$
\begin{aligned}
& \widetilde{\mathcal{S}}_{\alpha \beta}\left(\tau_{1}-\tau_{2}\right)=\widetilde{\mathcal{S}}_{\alpha \beta}\left(\tau_{1}, \tau_{2}\right) \\
& \quad=-\left\langle\mathbb{T}_{\tau} \Psi_{\alpha}\left(\tau_{1}\right) \Psi_{\beta}^{\dagger}\left(\tau_{2}\right)\right\rangle, \\
& \widetilde{\mathcal{D}}\left(k, k^{\prime}, \tau_{1}-\tau_{2}\right)=\widetilde{\mathcal{D}}\left(k, k^{\prime}, \tau_{1}, \tau_{2}\right) \\
& \quad=-\left\langle\mathbb{T}_{\tau} \Phi\left(k, \tau_{1}\right) \Phi\left(k^{\prime}, \tau_{2}\right)\right\rangle .
\end{aligned}
$$

The temperature propagators are functions of the difference of their arguments only. Therefore, according to the restriction (24) they are defined on the finite range of length $2 \beta$. Moreover, they fulfill the symmetric boundary condition $\widetilde{\mathcal{G}}(-\beta)=\widetilde{\mathcal{G}}(\beta)$. Therefore one can represent them as the following Fourier series [7-10]:

$$
\widetilde{\mathcal{G}}(\tau)=\frac{1}{\beta} \sum_{n=-\infty}^{\infty} \mathrm{e}^{-\mathrm{i} \omega_{n} \tau} \widetilde{\mathcal{G}}\left(\omega_{n}\right),
$$

where

$$
\omega_{n}=\left\{\begin{array}{cl}
\frac{2 n \pi}{\beta} & \text { bosons, } \\
\frac{2(n+1) \pi}{\beta} & \text { fermions. }
\end{array}\right.
$$

The Fourier coefficients $\widetilde{\mathcal{G}}\left(\omega_{n}\right)$ can be easily reconstructed from the inverse Fourier transform

$$
\widetilde{\mathcal{G}}\left(\omega_{n}\right)=\int_{0}^{\beta} \mathrm{d} \tau \mathrm{e}^{\mathrm{i} \omega_{n} \tau} \widetilde{\mathcal{G}}(\tau) .
$$

Since all properties of a two-level atom will be determined via the free temperature propagators we will now calculate them in detail. Similarly as it was done at zero temperature [1], free propagators are defined in terms of the field operators in the Matsubara-Dirac picture

$$
\begin{aligned}
& \widetilde{S}_{\alpha \beta}\left(\tau_{1}, \tau_{2}\right)=-\left\langle\mathbb{T}_{\tau} \psi_{\alpha}\left(\tau_{1}\right) \psi_{\beta}^{\dagger}\left(\tau_{2}\right)\right\rangle_{0}, \\
& \widetilde{D}\left(k, k^{\prime}, \tau_{1}, \tau_{2}\right)=-\left\langle\mathbb{T}_{\tau} \phi\left(k, \tau_{1}\right) \phi\left(k^{\prime}, \tau_{2}\right)\right\rangle_{0} .
\end{aligned}
$$

To find the free temperature electron propagator one has to use the averages of the bilinear combinations of the fermionic field operators over the statistical assembly. Then by simple calculations one finds the following representation of the electron propagator:

$$
\begin{aligned}
& \widetilde{S}\left(\tau_{1}, \tau_{2}\right)=-\left(\frac{\theta\left(\tau_{1}-\tau_{2}\right)}{1+\mathrm{e}^{-m \beta \sigma_{z}}}-\frac{\theta\left(\tau_{1}-\tau_{2}\right)}{1+\mathrm{e}^{m \beta \sigma_{z}}}\right) \\
& \quad \times \mathrm{e}^{-\left(m \sigma_{z}\right)\left(\tau_{1}-\tau_{2}\right)} .
\end{aligned}
$$

Consequently, the Fourier coefficient of this propagator reads

$$
\widetilde{S}\left(\omega_{n}\right)=\frac{1}{\mathrm{i} \omega_{n}-m \sigma_{z}}, \quad \text { where } \quad \omega_{n}=\frac{(2 n+1) \pi}{\beta} .
$$

Similarly, by using appropriate averages of bosonic field operators, one can get the free propagator of the electromagnetic field. It is not hard task to find the 
free temperature photon propagator and to show that its Fourier component reads

$$
\widetilde{D}\left(k, k^{\prime}, \omega_{n}\right)=-\frac{\delta\left(k-k^{\prime}\right)}{\omega_{n}^{2}+k^{2}}, \quad \text { where } \quad \omega_{n}=\frac{2 n \pi}{\beta} .
$$

4.2. Perturbation series and Feynman rules

The methods of quantum field theory at finite temperature are based on theorems which are formally very similar to the standard theorems used at zero temperature. This observation gives us a possibility to adapt automatically all well known machinery to this more complicated physical situation.

The main relation which gives us a chance to start perturbation calculations is a generalized Gell-Mann and Low theorem. It gives us explicitly a connection between the temperature correlation functions in the MatsubaraHeisenberg picture and the corresponding correlation functions in the Matsubara-Dirac picture. This fundamental relation is $[7,8]$ :

$$
\begin{aligned}
& \left\langle\mathbb{T}_{\tau} \mathcal{O}_{1}\left(\tau_{1}\right) \ldots \mathcal{O}_{n}\left(\tau_{n}\right)\right\rangle \\
& =\frac{\left\langle\mathbb{T}_{\tau} O_{1}\left(\tau_{1}\right) \ldots O_{n}\left(\tau_{n}\right) \mathrm{e}^{-\int_{0}^{\beta} \mathrm{d} \tau \mathcal{H}_{\mathrm{I}}(\tau)}\right\rangle_{0}}{\left\langle\mathbb{T}_{\tau} \mathrm{e}^{-\int_{0}^{\beta} \mathrm{d} \tau \mathcal{H}_{\mathrm{I}}(\tau)}\right\rangle_{0}} .
\end{aligned}
$$

One can easily check that the formal structure of this relation corresponds to the structure of the corresponding equation at zero temperature [1]. Since the integration on the right hand side is done only over the range $[0, \beta]$, now it is clear why we restricted in (24) the imaginary-time parameters to this range. Formal expansion of the right hand side in the powers of interaction Hamiltonian leads us directly to the concept of the Feynman diagrams which are just simple graphical representations of the appropriate elements of the perturbation series. As usual, any temperature correlation function in the MatsubaraHeisenberg picture can be expressed as a infinite sum of connected Feynman diagrams.

The finite-temperature Feynman rules for studied qubit system are very simple. At first one should draw Feynman diagrams according to the same rules as were used at zero temperature [1]. Then one assigns them corresponding mathematical expressions. It can be done in the frequency domain by applying following rules:

- Each photon line between any two vertices represents the free temperature photon propagator

$$
-\widetilde{D}\left(k, k^{\prime}, \omega_{n}\right)=\frac{\delta\left(k-k^{\prime}\right)}{\omega_{n}^{2}+k^{2}},
$$

where $\omega_{n}=\frac{2 n \pi}{\beta}$.

- Each fermion line between any two vertices represents the free temperature fermion propagator

$$
-\widetilde{S}\left(\omega_{n}\right)=\frac{-1}{\mathrm{i} \omega_{n}-m \sigma_{z}},
$$

where $\omega_{n}=\frac{(2 n+1) \pi}{\beta}$.

- Each vertex represents the expression

$$
-V(k)=-g(k) \sigma_{x} \text {. }
$$

- In each vertex the frequency conservation law holds. It is a consequence of the imaginary time translation invariance of the system. This rule is a counterpart of the energy conservation law at zero temperature.

- The fermion propagators are $2 \times 2$ matrices and therefore they should be multiplied in the order given by the directions of the lines.

- Each closed fermion loop leads to the trace operation of the matrices and gives a factor -1 . This rule is a simple consequence of the fermionic nature of the electron field operators.

In the end one should integrate such an expression over all internal momenta $k$ and take a sum over all internal frequencies $\omega_{n}$. Moreover, each sum must be divided by $\beta$.

\section{Temperature photon propagator}

Now we will find the temperature photon propagator in the lowest order of perturbation theory. Since there exists a formal connection between all Feynman rules at finite and zero temperatures, we can automatically write down the fundamental relation between photon propagator in the Matsubara-Heisenberg and in the Matsubara-Dirac pictures. This relation reads [1]:

$$
\begin{aligned}
& \widetilde{\mathcal{D}}\left(k, k^{\prime}, \omega_{n}\right)=\widetilde{D}\left(k, k^{\prime}, \omega_{n}\right)+\int_{0}^{\infty} \mathrm{d} k_{1} \int_{0}^{\infty} \mathrm{d} k_{2} \\
& \quad \times \widetilde{D}\left(k, k^{\prime}, \omega_{n}\right) \widetilde{\Pi}\left(k_{1}, k_{2}, \omega_{n}\right) \widetilde{\mathcal{D}}\left(k_{2}, k^{\prime}, \omega_{n}\right) .
\end{aligned}
$$

We introduced here the so-called temperature self-energy function $\widetilde{\Pi}$ which is built from all irreducible connected Feynman diagrams with two external photon lines

$$
\begin{aligned}
& -\widetilde{\Pi}\left(k_{1}, k_{2}, \omega_{n}\right)=\bigcirc=\bigcirc=\bigcirc+\vartheta_{3}+\omega^{2} \\
& +\ldots
\end{aligned}
$$

One can transform the relation (33) to a more convenient form

$$
\begin{aligned}
\widetilde{\mathcal{D}}\left(k, k^{\prime}, \omega_{n}\right) & =-\frac{\delta\left(k-k^{\prime}\right)}{\omega_{n}^{2}+k^{2}} \\
& +\frac{g(k)}{\omega_{n}^{2}+k^{2}} \frac{1}{\widetilde{P}\left(\omega_{n}\right)^{-1}-\widetilde{h}\left(\omega_{n}\right)} \frac{g\left(k^{\prime}\right)}{\omega_{n}^{2}+k^{\prime 2}},
\end{aligned}
$$

where we introduced a new function

$$
\widetilde{h}\left(\omega_{n}\right)=-\int_{0}^{\infty} \mathrm{d} k \frac{g^{2}(k)}{\omega_{n}^{2}+k^{2}} .
$$

We also define the temperature transition matrix $\widetilde{T}$ :

$$
\begin{gathered}
-\widetilde{T}\left(\omega_{n}\right)=\frac{1}{\bigoplus^{-1}-\ldots} \\
=-\frac{1}{\widetilde{P}^{-1}\left(\omega_{n}\right)-\widetilde{h}\left(\omega_{n}\right)} .
\end{gathered}
$$

Now we are ready to find the lowest order correction to the temperature Feynman photon propagator. The temperature self-energy function of the photon in this 
order is represented by the following Feynman diagram:

$$
\begin{aligned}
& -\widetilde{P}^{(2)}\left(\omega_{n}\right)=\{ \\
& =-\frac{1}{\beta} \sum_{n^{\prime}} \operatorname{Tr}\left(\sigma_{x} \widetilde{S}\left(\omega_{n^{\prime}}+\omega_{n}\right) \sigma_{x} \widetilde{S}\left(\omega_{n^{\prime}}\right)\right) .
\end{aligned}
$$

The summation over $n^{\prime}$ can be easily done and the details of these calculations are presented in Appendix B. We rewrite a result (75) derived there as follows:

$$
\widetilde{P}^{(2)}\left(\omega_{n}\right)=-\frac{4 m}{4 m^{2}+\omega_{n}^{2}} \tanh \left(\frac{\beta m}{2}\right) .
$$

From Eq. (35) one can easily find the temperature transition matrix

$$
\widetilde{T}^{(2)}\left(\omega_{n}\right)=-\frac{4 m}{\left(4 m^{2}+\omega_{n}^{2}\right) \operatorname{coth}\left(\frac{\beta m}{2}\right)-4 m \widetilde{h}\left(\omega_{n}\right)}
$$

It is worth to notice that taking the zero-temperature limit $(\beta \rightarrow \infty)$ at this point of our analysis has no physical sense. This fact will be explained later.

\section{Real time propagators}

Let us now return to the problem of the linear polarizability of the atom and also introduce the so-called retarded photon propagator. For the operators in the Heisenberg picture this propagator is defined as follows:

$$
\mathcal{D}_{\mathrm{R}}\left(k, k^{\prime}, t, t^{\prime}\right)=-\mathrm{i} \theta\left(t-t^{\prime}\right)\left\langle\left[\Phi(t), \Phi\left(t^{\prime}\right)\right]\right\rangle
$$

and for the free photon field (in the interaction picture) it reads

$$
D_{\mathrm{R}}\left(k, k^{\prime}, t, t^{\prime}\right)=-\mathrm{i} \theta\left(t-t^{\prime}\right)\left\langle\left[\phi(t), \phi\left(t^{\prime}\right)\right]\right\rangle_{0} .
$$

There exists a general connection between the retarded photon propagator defined above and the polarizability of the atom given by (17). This connection follows from the equation of motion (13b), a definition of the polarizability (17), and the properties of the free retarded photon propagator $(39 \mathrm{~b})$. The leading argument is identical as at zero temperature and it was given by us in [1]. Therefore we will not repeat it here. This connection has a form

$$
\begin{gathered}
\mathcal{D}_{\mathrm{R}}\left(k, k^{\prime}, k_{0}\right)=D_{\mathrm{R}}\left(k, k^{\prime}, k_{0}\right)-\frac{1}{A} \int_{0}^{\infty} \mathrm{d} k_{1} g\left(k_{1}\right) \int_{0}^{\infty} \mathrm{d} k_{2} \\
\quad \times g\left(k_{2}\right) D_{\mathrm{R}}\left(k, k_{1}, k_{0}\right) \alpha\left(k_{0}\right) D_{\mathrm{R}}\left(k_{2}, k^{\prime}, k_{0}\right),
\end{gathered}
$$

where $\mathcal{D}_{\mathrm{R}}\left(k, k^{\prime}, k_{0}\right)$ and $D_{\mathrm{R}}\left(k, k^{\prime}, k_{0}\right)$ are the Fourier transforms of the retarded photon propagators defined by Eq. (39):

$$
\begin{aligned}
& \mathcal{D}_{\mathrm{R}}\left(k, k^{\prime}, k_{0}\right)=\int_{-\infty}^{\infty} \mathrm{d} t \mathrm{e}^{\mathrm{i} k_{0} t} \mathcal{D}_{\mathrm{R}}\left(k, k^{\prime}, t, 0\right), \\
& D_{\mathrm{R}}\left(k, k^{\prime}, k_{0}\right)=\int_{-\infty}^{\infty} \mathrm{d} t \mathrm{e}^{\mathrm{i} k_{0} t} D_{\mathrm{R}}\left(k, k^{\prime}, t, 0\right) .
\end{aligned}
$$

From Eq. (40) it clearly follows that we need an appropriate retarded photon propagator to find the polarizability of a two-level atom (or more generally — the linear response of the system).

\subsection{Spectral representation of propagators}

Now we will demonstrate that there exists an exact mathematical connection between the real time and the temperature propagators. For future benefits we restrict ourself just to the retarded propagator of the electromagnetic field. Other propagators, like the advanced propagator or the chronologically ordered propagator, can be also reconstructed from the temperature propagators [7-10] but in the case of the linear polarizability of the atom they are not interesting.

It is worthwhile to notice that this general connection between different types of propagators is related to the problem of two different views on interactions between qubit and the electromagnetic field: the scattering view, when we control the initial and final states of the photon, and the linear response view, when we control only the initial state and we perform summation over all final states. This observation settles the "sign-prescription" controversy [1, 23-29, 34]. Moreover, all these arguments can be also extended to the nonlinear wave mixing [35].

From Eq. (27) one can find the Fourier transform of the temperature photon propagator defined by (25b)

$$
\begin{aligned}
& \widetilde{\mathcal{D}}\left(k, k^{\prime}, \omega_{n}\right)=\int_{0}^{\beta} \mathrm{d} \tau \mathrm{e}^{\mathrm{i} \omega_{n} \tau} \widetilde{\mathcal{D}}\left(k, k^{\prime}, \tau, 0\right)=\int_{0}^{\beta} \mathrm{d} \tau \\
& \quad \times \frac{\mathrm{e}^{\mathrm{i} \omega_{n} \tau}}{\mathcal{Z}} \sum_{n, m}\left\langle n\left|\mathrm{e}^{-\beta \mathcal{K}} \mathrm{e}^{\mathcal{K} \tau} \Phi(k) \mathrm{e}^{-\mathcal{K} \tau}\right| m\right\rangle\left\langle m\left|\Phi\left(k^{\prime}\right)\right| n\right\rangle \\
& =\sum_{n, m} \int_{0}^{\beta} \mathrm{d} \tau \mathrm{e}^{\left(\mathrm{i} \omega_{n}+K_{n}-K_{m}\right) \tau} \frac{\mathrm{e}^{-\beta K_{n}}}{\mathcal{Z}} \\
& \quad \times\langle n|\Phi(k)| m\rangle\left\langle m\left|\Phi\left(k^{\prime}\right)\right| n\right\rangle .
\end{aligned}
$$

In this sequence of equalities we have used the set $\{|n\rangle\}$ of the eigenvectors of the statistical Hamiltonian $\mathcal{K}$ with the eigenvalues $K_{n}$. After performing an integration over $\tau$, one finds

$$
\widetilde{\mathcal{D}}\left(k, k^{\prime}, \omega_{n}\right)=\int_{-\infty}^{\infty} \mathrm{d} M \frac{\mathfrak{M}\left(M, k, k^{\prime}\right)}{\mathrm{i} \omega_{n}-M},
$$

where the temperature spectral matrix of the propagator is given by

$$
\begin{aligned}
& \mathfrak{M}\left(M, k, k^{\prime}\right)=\sum_{n, m} \delta\left(M+K_{n}-K_{m}\right) \frac{\mathrm{e}^{-\beta K_{m}}-\mathrm{e}^{-\beta K_{n}}}{\mathcal{Z}} \\
& \quad \times\langle n|\Phi(k)| m\rangle\left\langle m\left|\Phi\left(k^{\prime}\right)\right| n\right\rangle .
\end{aligned}
$$

In an analogous way one can show that retarded propagator (41a) has the following spectral representation:

$$
\begin{aligned}
\mathcal{D}_{\mathrm{R}} & \left(k, k^{\prime}, t, 0\right) \\
& =-\mathrm{i} \theta(t)\left[\left\langle\Phi(k, t) \Phi\left(k^{\prime}\right)\right\rangle-\left\langle\Phi\left(k^{\prime}\right) \Phi(k, t)\right\rangle\right] \\
& =\int_{-\infty}^{\infty} \frac{\mathrm{d} k_{0}}{2 \pi} \mathrm{e}^{-\mathrm{i} k_{0} t} \int_{-\infty}^{\infty} \mathrm{d} M \frac{\mathfrak{M}\left(M, k, k^{\prime}\right)}{k_{0}-M+\mathrm{i} \epsilon} .
\end{aligned}
$$

It means that the Fourier transform of the retarded photon propagator reads

$$
\mathcal{D}_{\mathrm{R}}\left(k, k^{\prime}, k_{0}\right)=\int_{-\infty}^{\infty} \mathrm{d} M \frac{\mathfrak{M}\left(M, k, k^{\prime}\right)}{k_{0}-M+\mathrm{i} \epsilon} .
$$




\subsection{The analytic continuation}

The spectral representation of the propagators is very useful to find the connection between different types of propagators in the frequency domain. First, let us notice that the retarded propagator (46) treated as a function of the continuous complex variable $k_{0}$ is analytic in the upper half of the complex plane. It means that if one defines the function $F(z)$ of the complex variable $z$,

$$
F(z)=\int_{-\infty}^{\infty} \mathrm{d} M \frac{\mathfrak{M}\left(M, k, k^{\prime}\right)}{z-M},
$$

then this function in the upper half-plane would be exactly equal to the retarded photon propagator (46). Additionally, at points $z_{n}=\mathrm{i} \omega_{n}$ this function is equal to the Fourier components of the temperature photon propagator (43). Therefore the temperature photon propagator for positive $\omega_{n}$ can be easily reconstructed directly from the retarded propagator in the following way:

$$
\widetilde{\mathcal{D}}\left(k, k^{\prime}, \omega_{n}\right)=\mathcal{D}_{\mathrm{R}}\left(k, k^{\prime}, \mathrm{i} \omega_{n}\right) .
$$

The Fourier components for the negative frequencies can be reconstructed as well because the following relation holds:

$$
\widetilde{\mathcal{D}}\left(k, k^{\prime},-\omega_{n}\right)=\widetilde{\mathcal{D}}^{*}\left(k, k^{\prime}, \omega_{n}\right) .
$$

The inverse problem, how to construct the retarded propagator from the temperature propagator, is more complicated. The reason is that the Fourier components of the temperature propagator are defined only on a discrete set of frequencies $\omega_{n}$. Therefore, there exist many analytical continuations of the temperature propagator to the whole complex plane and one needs to add other conditions to choose an appropriate continuation. This general problem was discussed in the past $[7,8,10,36]$ and now it is known how to manage it. In our case the retarded propagator can be constructed with the following rule:

$$
\mathcal{D}_{\mathrm{R}}\left(k, k^{\prime}, k_{0}\right)=F\left(-\mathrm{i} k_{0}+\epsilon\right), \quad \text { for } \quad k_{0}>0 .
$$

To find the retarded propagator for the negative $k_{0}$ one has to use the crossing relation given in our previous paper [1].

\section{Polarizability of the two-level atom}

In the previous section we have found a connection between the temperature photon propagator and the retarded photon propagator. It was very important step because it gives us a possibility to find the temperature properties of the linear polarizability of the atom. To make it obvious let us recall once more fundamental formulae for different types of the photon propagators in the theory with interactions

$$
\begin{aligned}
& \widetilde{\mathcal{D}}\left(k, k^{\prime}, \omega_{n}\right)=\widetilde{D}\left(k, k^{\prime}, \omega_{n}\right) \\
& \quad+\frac{g(k)}{k^{2}+\omega_{n}^{2}} \widetilde{T}\left(\omega_{n}\right) \frac{g\left(k^{\prime}\right)}{k^{2}+\omega_{n}^{2}}, \\
& \mathcal{D}_{\mathrm{R}}\left(k, k^{\prime}, k_{0}\right)=D_{\mathrm{R}}\left(k, k^{\prime}, k_{0}\right) \\
& \quad-\frac{1}{A} \frac{g(k)}{k^{2}-\left(k_{0}-\mathrm{i} \epsilon\right)^{2}} \alpha\left(k_{0}\right) \frac{g\left(k^{\prime}\right)}{k^{2}-\left(k_{0}-\mathrm{i} \epsilon\right)^{2}} .
\end{aligned}
$$

For positive $k_{0}$ we can reconstruct the retarded propagator (51b) from the temperature one (51a) by making the substitution $\omega_{n} \rightarrow-\mathrm{i} k_{0}+\epsilon$. Therefore, it is self-evident that the linear polarizability $\alpha\left(k_{0}\right)$ can be extracted from the temperature transition matrix $\widetilde{T}\left(k_{0}\right)$. The rule is the following:

$$
\alpha\left(k_{0}\right)=-A \widetilde{T}\left(-\mathrm{i} k_{0}+\epsilon\right), \quad \text { for } \quad k>0 .
$$

Once we have determined the temperature transition matrix in the second order of perturbation (38), we are able to find the polarizability in this order. For positive $\omega$ one gets

$$
\begin{aligned}
& \alpha^{(2)}(\omega)=4 m A /\left\{\left(4 m^{2}-\omega^{2}\right) \operatorname{coth}\left(\frac{\beta m}{2}\right)\right. \\
& -4 m[\Delta(\omega)+\mathrm{i} \Gamma(\omega)]\} .
\end{aligned}
$$

Functions $\Delta(\omega)$ and $\Gamma(\omega)$ are defined as real and imaginary part of the function $h(\omega)=\widetilde{h}(-\mathrm{i} \omega+\epsilon)$ :

$$
\begin{aligned}
& \Delta(\omega)=\wp \int_{0}^{\infty} \mathrm{d} k \frac{g^{2}(k)}{k^{2}-\omega^{2}}, \\
& \Gamma(\omega)=\frac{\pi}{2} \frac{g^{2}(\omega)}{|\omega|} .
\end{aligned}
$$

Finally, using the crossing symmetry of the propagators (49), we get

$$
\begin{aligned}
& \alpha^{(2)}(\omega)=4 m A /\left\{\left(4 m^{2}-\omega^{2}\right) \operatorname{coth}\left(\frac{\beta m}{2}\right)\right. \\
& -4 m[\Delta(\omega)+i \operatorname{sgn}(\omega) \Gamma(\omega)]\} .
\end{aligned}
$$

The last step is to use the correspondence formula (16) to find a real physical atom polarizability. To do so one has to find a ratio of the partition functions $\mathcal{Z} / \widehat{\mathcal{Z}}$. In the lowest order of perturbation it is given by the formula (70):

$$
\frac{\mathcal{Z}}{\widehat{\mathcal{Z}}} \approx \frac{\tanh (\beta m)}{\tanh \left(\frac{\beta m}{2}\right)}\left[1+\left(1-\frac{\tanh (\beta m)}{\tanh \left(\frac{\beta m}{2}\right)}\right) \delta^{(2)}\right] .
$$

This all means that in the lowest order of perturbation theory the Fourier transform of the linear polarizability of a two-level atom has the form

$$
\begin{aligned}
& \mathfrak{a}^{(2)}(\omega)=4 m A_{\mathrm{T}} /\left\{4 m^{2}-\omega^{2}\right. \\
& \left.-4 m\left[\Delta_{\mathrm{T}}(\omega)+\operatorname{isgn}(\omega) \Gamma_{\mathrm{T}}(\omega)\right]\right\},
\end{aligned}
$$

where we introduced the effective temperature-dependent amplitude of the polarizability, as well as an effective shift and width of the resonance

$$
\begin{aligned}
& A_{\mathrm{T}}=A \tanh (\beta m) \\
& \times\left[1+\left(1-\frac{\tanh (\beta m)}{\tanh \left(\frac{\beta m}{2}\right)}\right) \delta^{(2)}\right], \\
& \Delta_{\mathrm{T}}(\omega)=\Delta(\omega) \tanh \left(\frac{\beta m}{2}\right), \\
& \Gamma_{\mathrm{T}}(\omega)=\Gamma(\omega) \tanh \left(\frac{\beta m}{2}\right) .
\end{aligned}
$$

It is very simple now to verify that in the low temperature limit $(\beta \rightarrow \infty)$ we reconstruct the polarizability at 
zero temperature given by us before in [1]. Moreover, we find that our result predicts a small correction to the behavior of the amplitude of the linear polarizability, well known from the theory of the magnetic resonance [37]. This correction is a second-order outcome of the interaction and in the limit when $g(k) \rightarrow 0$ we reconstruct the previous result.

All physical properties of the linear polarizability of an atom at finite temperature directly follow from Eq. (56). As it is seen, the magnitudes of the resonance shift and the width and the amplitude of the polarizability decrease when the temperature grows. It means that the capabilities of keeping the qubit under control at high temperature are extremely poor, since the reaction of the system to the external electromagnetic field is weak (small amplitude), and the resonance is narrow (small resonance width).

\section{Thermal line narrowing}

Notice that temperature dependence of the relative shift $\Delta_{\mathrm{T}} / \Delta$ and the relative width $\Gamma_{\mathrm{T}} / \Gamma$ of the resonance in the lowest order of perturbation do not depend on the function $g(k)$ but they depend only on the energy gap between the qubit states $\Delta E=2 m$ (Fig. 1). This means that it is a universal property of physical qubits - it does not depend on the details of experimental qubit realizations encoded in the coupling function $g(k)$. Moreover, the interaction between qubits and the quantized electromagnetic field is very similar to the interaction with the quantized vibrations of the crystal lattice (phonons). Therefore, one can imagine that the dependence on temperature in such a case would be very similar. This means that temperature dependence of the relative shift and the relative width of the resonance is universal for all qubits with frozen spatial degrees of freedom.

From Eq. (57c) one can find the sensitivity of the lifetime of the excited state (the inverse of $\Gamma$ ) to the temperature for different experimental realizations of qubits. We define the characteristic temperature $T_{\mathrm{C}}$ as the temperature at which the lifetime of the excited state becomes ten times longer than at zero temperature. One can find that it depends only on the energy gap of the two-level atom

$$
T_{\mathrm{C}} \approx 2.5 \times \frac{\Delta E}{k} .
$$

The comparison of the characteristic temperature for different realizations is given in Table. Obviously one should remember that treating a given system as a two-level atom often is just an approximation. Therefore, anytime when this approximation is made, one should check if it is valid. However, there are real two-level system in nature: spin- $1 / 2$ system and an electron in appropriate prepared quantum dot. In addition, treating fine splitting levels of atomic states as a two-level system at low temperatures seems to be a good approximation.

In conclusion, let us concentrate on the problem of the width of the resonance. As was shown above, the

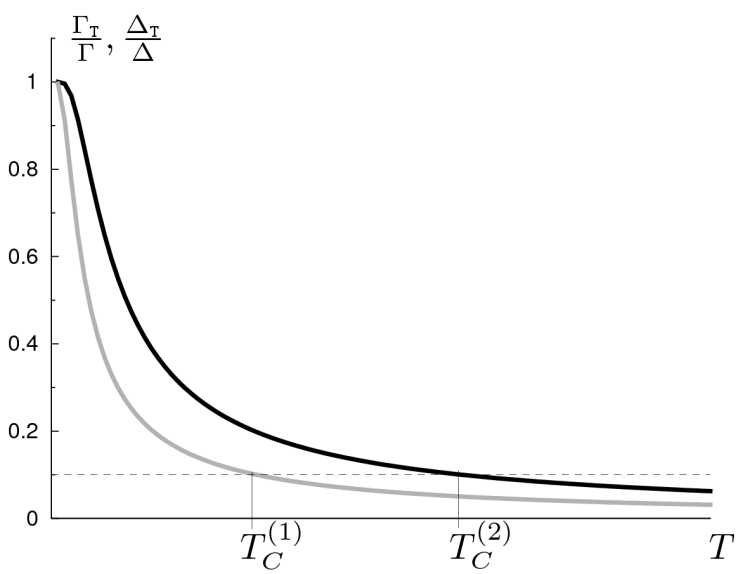

Fig. 1. Temperature dependence of the relative shift and relative width of the resonance for two qubits with different energy gap. Energy gap $\Delta E^{(1)}$ for the first qubit (grey line) is smaller than energy gap $\Delta E^{(2)}$ for the second qubit (black line). Therefore, the characteristic temperature $T_{\mathrm{C}}$ is smaller in the first case than in the second case $\left(T_{\mathrm{C}}^{(1)}<T_{\mathrm{C}}^{(2)}\right)$.

TABLE

Comparison of the characteristic temperature $T_{\mathrm{C}} \approx 2.5 \times \frac{\Delta E}{k}$ (temperature at which the lifetime of the excited state becomes ten times longer than at zero temperature) for different physical realizations of qubits [38-43].

\begin{tabular}{c|c|c}
\hline \hline Qubit realization & $\Delta E=2 m$ & $T_{\mathrm{C}}$ \\
\hline electron in quantum dot & $\approx 50 \mathrm{meV}$ & $\approx 1500 \mathrm{~K}$ \\
${ }^{2} S_{1 / 2}$ splitting in ${ }^{199} \mathrm{Hg}^{+}$ & $167 \mu \mathrm{eV}$ & $\approx 4.8 \mathrm{~K}$ \\
${ }^{2} S_{1 / 2}$ splitting in ${ }^{113} \mathrm{Cd}^{+}$ & $63 \mu \mathrm{eV}$ & $\approx 1.8 \mathrm{~K}$ \\
${ }^{2} S_{1 / 2}$ splitting in ${ }^{87} \mathrm{Rb}^{+}$ & $28 \mu \mathrm{eV}$ & $\approx 0.8 \mathrm{~K}$
\end{tabular}

resonance width decreases unexpectedly when the temperature grows. Such a behavior is counterintuitive. In typical situations we expect broadening of the resonance rather than narrowing. The best known examples where the resonance broadens with temperature are obviously the heated gases where, due to the Doppler effect caused by the thermal motion of the molecules, all spectral lines become broader when the temperature grows.

It is instructive to notice that the main result of this paper deals with old problem of the shapes of the resonances in two-level systems and brings some new arguments to the discussion about validity of approximate methods [44-47]. For example, in the paper by Beck et al. [48] the authors find different dependence of the relative width of the resonance and predict broadening of the spectral line due to the Boltzmann factor $\operatorname{coth}(\beta m)$ rather than narrowing predicted by us in $(57 \mathrm{c})$. Nevertheless, it should be emphasized that the result given in [48] is obtained by making many non-obvious approximations. The most problematic one is the validity of 
the usually used "decoupling approximation". As was noticed in [46] this approximation is not based on any perturbation arguments and for that it is to a certain extent arbitrary. Therefore, one cannot check the credibility of the result at low temperature. It is also known that it is not certain that the result of Beck et al. is valid in the situation when the spectral density of bosons at zero frequency is zero [44]. Moreover, as was mentioned in [47], the decoupling approximation ignores the fact that for high frequencies the two-level system will shake-off its polarization cloud. In consequence, to make a theory self-consistent, one needs to introduce high-frequency cut-off. Obviously, this is a serious problem in a weak coupling regime. We resolve it by making the renormalization.

In contrast to all previous works, the theory presented in this paper is based on sound first principles. There are no additional assumptions made and all results are derived from the well defined model. The only assumption we made is on the validity of the perturbation expansion in the coupling between two-level system and the electromagnetic field. All remaining steps are just a simple consequence of this assumption and they are obtained by clear mathematical arguments. Presented technique is based on the general principles of the thermal quantum field theory methods which has brought many fundamental results in many-body problems. Even if one questions generality of this assumption, it is obvious that the result (56) in the weak coupling limit is correct. Since the characteristic temperature $T_{\mathrm{C}}$ of the narrowing of the resonance (58) is of the order of the energy gap $2 m$ and the result obtained by Beck et al. is valid only at high temperatures (when $k T \gg 2 m$ ) one should expect that the narrowing of the resonance predicted by us is significant and it should be experimentally recognizable.

The description of two-level systems in terms of temperature propagators and their analytical properties was discussed before [49], but it was done without introducing the creation and annihilation operators for electron states. Therefore, the whole analysis was carried out only in the effective fermion propagator approximation. The full potential of this formulation with systematic use of the Matsubara-Feynman diagrams has not been exploited.

There exist some theoretical predictions in the literature on the narrowing of the resonance known as the phenomenon of motional narrowing. First theoretical analysis of the motional narrowing was given in the fundamental paper on nuclear resonance by Bloembergen et al. [50]. The narrowing of the resonance is explained as due to the influence of the thermal motion on the strength of the spin-spin interactions. It turns out that the thermal motion effectively leads to the decrease of the damping effects induced by such interactions. The general problem of the motional narrowing was studied and generalized in the language of stochastic processes under the assumption that the system is subjected to a fluctuating environment [51]. There exist many physical systems where the motional narrowing is found [52-55].

The origin of the narrowing of the resonance predicted in presented paper is completely different. Notice that in the studied situation there is only one frozen qubit. Therefore there is no place for any influence of the Doppler effect or of any interaction with other qubits. This suggests that it should be called motionless narrowing rather than the motional one, since it is clear that freezing of spatial degrees of freedom is a key element of the line narrowing.

In the end let us give some intuitive arguments and give the microscopic explanation of the narrowing of the resonance for one frozen qubit. When the temperature of the thermostat is different from zero, a two-level atom is incessantly stricken by the thermal photons and obviously the number of resonant photons increases with the temperature. However, this has no influence on the atom when it is excited, since the spontaneous emission does not depend on the temperature and at small temperatures it always dominates the stimulated emission. Strictly speaking, the emission from the excited state is almost identical as at $T=0$. Nevertheless, the influence of the resonant photons is significant when the atom is in the ground state because the probability of an excitation of the atom increases with temperature. A combination of these two effects - the spontaneous emission (independent of the temperature) and the stimulated absorption (highly dependent of the temperature) leads effectively to the prolonged atom lifetime in the excited state. It means that the width of the resonance shape becomes smaller.

\section{Acknowledgments}

I thank Professor Iwo Białynicki-Birula for his incisive questions, useful comments, and fruitful discussion. This work was supported by a grant from the Polish Ministry of Science and Higher Education.

\section{Appendix A \\ Relations between partition functions}

In this appendix we derive direct relations between the partition functions $\mathcal{Z}$ and $\widehat{\mathcal{Z}}$. These relations are exact. They hold in any order of perturbation theory.

\section{A.1. Difference of partition functions}

Let us consider a family of the systems parameterized by $\lambda$ described by the following statistical Hamiltonian:

$$
\widehat{\mathcal{K}}_{\lambda}=\widehat{\mathcal{K}}_{0}+\lambda \widehat{\mathcal{H}}_{\mathrm{I}}
$$

and assume that each of those systems is in the quantum state described by a density matrix

$$
\widehat{\rho}_{\lambda}=\mathrm{e}^{-\beta \widehat{\mathcal{K}}_{\lambda}}, \quad \widehat{\mathcal{Z}}_{\lambda}=\operatorname{Tr}\left(\mathrm{e}^{-\beta \widehat{\mathcal{K}}_{\lambda}}\right) .
$$

It is clear that for $\lambda=1$ we recover the situation described by the formulae (4) and for $\lambda=0$ by the formulae (6). Let us also introduce the second-quantized counterpart of this family 


$$
\begin{aligned}
& \mathcal{K}_{\lambda}=\mathcal{K}_{0}+\lambda \mathcal{H}_{\mathrm{I}}, \\
& \rho_{\lambda}=\mathrm{e}^{-\beta \mathcal{K}_{\lambda}}, \quad \mathcal{Z}_{\lambda}=\operatorname{Tr}\left(\mathrm{e}^{-\beta \mathcal{K}_{\lambda}}\right) .
\end{aligned}
$$

One can easily get a derivative of the partition function with respect to $\lambda$ for these two families

$$
\begin{aligned}
& \frac{\partial \mathcal{Z}_{\lambda}}{\partial \lambda}=-\beta \operatorname{Tr}\left(\mathrm{e}^{-\beta \mathcal{K}_{\lambda}} \mathcal{H}_{\mathrm{I}}\right)=-\frac{\beta}{\lambda} \mathcal{Z}_{\lambda}\left\langle\lambda \mathcal{H}_{\mathrm{I}}\right\rangle_{\lambda}, \\
& \frac{\partial \widehat{\mathcal{Z}}_{\lambda}}{\partial \lambda}=-\beta \operatorname{Tr}\left(\mathrm{e}^{-\beta \widehat{\mathcal{K}}_{\lambda}} \widehat{\mathcal{H}}_{\mathrm{I}}\right)=-\frac{\beta}{\lambda} \widehat{\mathcal{Z}}_{\lambda}\left\langle\left\langle\lambda \widehat{\mathcal{H}}_{\mathrm{I}}\right\rangle\right\rangle_{\lambda} .
\end{aligned}
$$

The argument leading to the formula (16) is also valid for the systems with any $\lambda$. Since the interaction Hamiltonian $\mathcal{H}_{\mathrm{I}}$ has the needed property (in the qubit subspace it is represented by a traceless matrix proportional to the Pauli $\sigma_{x}$ matrix), one has the important relation

$$
\left\langle\left\langle\lambda \widehat{\mathcal{H}}_{\mathrm{I}}\right\rangle\right\rangle_{\lambda}=\frac{\mathcal{Z}_{\lambda}}{\widehat{\mathcal{Z}}_{\lambda}}\left\langle\lambda \mathcal{H}_{\mathrm{I}}\right\rangle_{\lambda} \text {. }
$$

This immediately leads us to the nontrivial equality of the derivatives

$$
\frac{\partial \mathcal{Z}_{\lambda}}{\partial \lambda}=\frac{\partial \widehat{\mathcal{Z}}_{\lambda}}{\partial \lambda}
$$

Therefore one gets a crucial relation

$$
\mathcal{Z}-\mathcal{Z}_{0}=\widehat{\mathcal{Z}}-\widehat{\mathcal{Z}}_{0} \text {. }
$$

It means that the difference between the partition functions $\mathcal{Z}$ and $\widehat{\mathcal{Z}}$ is the same as difference between the partition functions for noninteracting systems $\mathcal{Z}_{0}$ and $\widehat{\mathcal{Z}}_{0}$, which can be calculated directly.

\section{A.2. Ratio of partition functions}

From the general rules of the thermal quantum field theory we know that the partition function $\mathcal{Z}$ can be expressed as a sum of all vacuum-like Feynman diagrams

$$
\mathcal{Z}=\mathcal{Z}_{0}(1+\delta)
$$

where

$$
-\delta=(2)+\sqrt[2]{2}+m+\infty m \ldots
$$

With Eq. (65) one can find that

$$
\widehat{\mathcal{Z}}=\widehat{\mathcal{Z}}_{0}+\mathcal{Z}-\mathcal{Z}_{0}=\widehat{\mathcal{Z}}_{0}+\mathcal{Z}_{0} \delta
$$

and therefore the ratio of the partition functions is given by

$$
\frac{\mathcal{Z}}{\widehat{\mathcal{Z}}}=\frac{\mathcal{Z}_{0}}{\widehat{\mathcal{Z}}_{0}} \frac{1+\delta}{1+\frac{\mathcal{Z}_{0}}{\widehat{\mathcal{Z}}_{0}} \delta} .
$$

This result is correct in any order of perturbation theory and it is easy to verify that the ratio $\mathcal{Z}_{0} / \widehat{\mathcal{Z}}_{0}$ of the partition functions for non-iteracting systems reads

$$
\frac{\mathcal{Z}_{0}}{\widehat{\mathcal{Z}}_{0}}=\frac{\mathrm{e}^{-\beta m}+\mathrm{e}^{\beta m}+2}{\mathrm{e}^{-\beta m}+\mathrm{e}^{\beta m}}=\frac{\tanh (\beta m)}{\tanh \left(\frac{\beta m}{2}\right)} .
$$

To find an appropriate formula in a given order of perturbation one should expand the relation (68) in powers of the coupling constant (the form factor $g(k)$ ) and truncate the series at the appropriate position. For example, in the lowest (second) order one finds

$$
\frac{\mathcal{Z}}{\widehat{\mathcal{Z}}} \approx \frac{\mathcal{Z}_{0}}{\widehat{\mathcal{Z}}_{0}}\left[1+\left(1-\frac{\mathcal{Z}_{0}}{\widehat{\mathcal{Z}}_{0}}\right) \delta^{(2)}\right],
$$

where $\delta^{(2)}$ is represented only by one Feynman diagram (76a) and it is given by (79) evaluated in Appendix B.

\section{Appendix B \\ Second order calculations \\ B.1. Photon self-energy function}

The temperature self-energy function of the photon in the lowest order of perturbation theory is represented by the following Feynman diagram:

$$
\begin{aligned}
& -\widetilde{P}^{(2)}\left(\omega_{n}\right)=\{ \\
& =-\frac{1}{\beta} \sum_{n^{\prime}} \operatorname{Tr}\left(\sigma_{x} \widetilde{S}\left(\omega_{n^{\prime}}+\omega_{n}\right) \sigma_{x} \widetilde{S}\left(\omega_{n^{\prime}}\right)\right) .
\end{aligned}
$$

Depending on the quantum statistics (see Eq. (26)), the frequencies $\omega_{n}$ and $\omega_{n^{\prime}}$ are

$$
\omega_{n}=\frac{2 n \pi}{\beta}, \quad \omega_{n^{\prime}}=\frac{\left(2 n^{\prime}+1\right) \pi}{\beta} .
$$

One can easily transform the relation (71a) to a simpler form

$$
\begin{aligned}
& \widetilde{P}^{(2)}\left(\omega_{n}\right)=\frac{1}{\beta} \sum_{n^{\prime}=-\infty}^{\infty}\left[\frac{1}{\mathrm{i} \omega_{n^{\prime}}+\mathrm{i} \omega_{n}+m} \frac{1}{\mathrm{i} \omega_{n^{\prime}}-m}\right. \\
& \left.+\frac{1}{\mathrm{i} \omega_{n^{\prime}}+\mathrm{i} \omega_{n}-m} \frac{1}{\mathrm{i} \omega_{n^{\prime}}+m}\right] .
\end{aligned}
$$

The summation over $n^{\prime}$ can be done with the exploitation of the following mathematical identity:

$$
\begin{aligned}
& \sum_{n^{\prime}} \frac{1}{\mathrm{i} \omega_{n^{\prime}}-a} \frac{1}{\mathrm{i} \omega_{n^{\prime}}-b} \\
& \quad=-\frac{\beta}{2} \frac{\tanh \left(\frac{\beta b}{2}\right)-\tanh \left(\frac{\beta a}{2}\right)}{b-a}
\end{aligned}
$$

which is valid for $\omega_{n^{\prime}}=\left(2 n^{\prime}+1\right) \pi / \beta$.

The temperature self-energy function of the photon in the lowest order of perturbation theory has the form

$$
\begin{gathered}
\widetilde{P}^{(2)}\left(\omega_{n}\right)=-\frac{1}{2}\left[\frac{\tanh \left(\frac{\beta m}{2}\right)+\tanh \left(\frac{\beta}{2}\left(m+\mathrm{i} \omega_{n}\right)\right)}{\mathrm{i} \omega_{n}+2 m}\right. \\
\left.-\frac{\tanh \left(\frac{\beta m}{2}\right)+\tanh \left(\frac{\beta}{2}\left(m-\mathrm{i} \omega_{n}\right)\right)}{\mathrm{i} \omega_{n}-2 m}\right]
\end{gathered}
$$

Conclusively, according to the periodicity of the hyperbolic function and the relation (71c), the temperature self-energy function has the form

$$
\widetilde{P}^{(2)}\left(\omega_{n}\right)=-\frac{4 m}{4 m^{2}+\omega_{n}^{2}} \tanh \left(\frac{\beta m}{2}\right) .
$$

\section{B.2. Correction to the partition function}

The first correction to the partition function of the interacting system is represented by the following Feynman diagram:

$$
\begin{aligned}
& -\delta^{(2)}= \\
& =\frac{1}{\beta^{2}} \sum_{n} \sum_{n^{\prime}} \int_{0}^{\infty} \mathrm{d} k \int_{0}^{\infty} \mathrm{d} k^{\prime} \widetilde{D}\left(k, k^{\prime}, \omega_{n}\right) \\
& \quad \times \operatorname{Tr}\left(V(k) \widetilde{S}\left(\omega_{n^{\prime}}+\omega_{n}\right) V\left(k^{\prime}\right) \widetilde{S}\left(\omega_{n^{\prime}}\right)\right) .
\end{aligned}
$$


Summations run over the boson-like $\omega_{n}$ and the fermion-like $\omega_{n^{\prime}}$ :

$$
\omega_{n}=\frac{2 n \pi}{\beta}, \quad \omega_{n^{\prime}}=\frac{\left(2 n^{\prime}+1\right) \pi}{\beta} .
$$

However, the summation over $n^{\prime}$ can be easily expressed by the photon self-energy function in the second order of perturbation theory (71a) evaluated before

$$
\begin{aligned}
& -\delta^{(2)}=-\frac{1}{\beta} \int_{0}^{\infty} \mathrm{d} k g^{2}(k) \sum_{n} \frac{\widetilde{P}^{(2)}\left(\omega_{n}\right)}{\omega_{n}^{2}+k^{2}} \\
& =\frac{4 m}{\beta} \tanh \left(\frac{\beta m}{2}\right) \int_{0}^{\infty} \mathrm{d} k g^{2}(k) \\
& \quad \times \sum_{n} \frac{1}{\omega_{n}^{2}+4 m^{2}} \frac{1}{\omega_{n}^{2}+k^{2}} .
\end{aligned}
$$

One can do the summation over $n$ similarly as in formula $(72)$. For the bosonic frequencies $\left(\omega_{n}=2 n \pi / \beta\right)$ the appropriate summation rule reads

$$
\begin{aligned}
& \sum_{n} \frac{1}{\mathrm{i} \omega_{n}-a} \frac{1}{\mathrm{i} \omega_{n}-b} \\
& \quad=-\frac{\beta}{2} \frac{\operatorname{coth}\left(\frac{\beta b}{2}\right)-\operatorname{coth}\left(\frac{\beta a}{2}\right)}{b-a}
\end{aligned}
$$

After performing the appropriate summations one gets the final expression for the first correction to the partition function

$$
\begin{gathered}
\delta^{(2)}=\tanh \left(\frac{\beta m}{2}\right) \int_{0}^{\infty} \mathrm{d} k \frac{g^{2}(k)}{k\left(k^{2}-4 m^{2}\right)} \\
\times\left[2 m \operatorname{coth}\left(\frac{\beta k}{2}\right)-k \operatorname{coth}(\beta m)\right] .
\end{gathered}
$$

At zero temperature limit $(\beta \rightarrow \infty)$, this correction is finite and negative. It is a simple exercise to find that in this limit it has a form

$$
\lim _{\beta \rightarrow \infty} \delta^{(2)}=-\int_{0}^{\infty} \mathrm{d} k \frac{g^{2}(k)}{k(2 m+k)} .
$$

It is worthwhile to notice that even near the resonance, when $k \approx 2 m$, the integral (79) is well defined, because the dangerous pole in the denominator is canceled by an appropriate term in the numerator of the expression.

\section{References}

[1] I. Białynicki-Birula, T. Sowiński, Phys. Rev. A 76, 062106 (2007).

[2] T. Matsubara, Prog. Theor. Phys. 14, 351 (1955).

[3] K.M. Watson, Phys. Rev. 103, 489 (1956).

[4] R. Kubo, J. Phys. Soc. Japan 12, 570 (1957).

[5] P.C. Martin, J. Schwinger, Phys. Rev. 115, 1342 (1959).

[6] J.M. Luttinger, Phys. Rev. 119, 1153 (1960).

[7] A.A. Abrikosov, L.P. Gorkov, I.E. Dzyaloshinski, Methods of Quantum Field Theory in Statistical Physics, Translated by R.A. Silverman, Prentice-Hall Inc., New Jersey 1963.

[8] A.L. Fetter, J.D. Walecka, Quantum Theory of Many-Particle Systems, McGraw-Hill, New York 1971.
[9] J.I. Kapusta, Finite Temperature Field Theory, Cambridge University Press, Oxford 1989.

[10] A. Das, Finite Temperature Field Theory, World Sci., Singapore 1997.

[11] S. Sachdev, Phys. Rev. A 29, 2627 (1984).

[12] S.M. Barnett, P.L. Knight, Phys. Rev. A 33, 2444 (1986).

[13] J. Salo, S.M. Barnett, S. Stenholm, Opt. Commun. 259, 772 (2006).

[14] M.P. Almeida, F. de Melo, M. Hor-Meyll, A. Salles, S.P. Walborn, P.H. Souto Ribeiro, L. Davidovich, Science 316, 579 (2007).

[15] J. Laurat, K.S. Choi, H. Deng, C.W. Chou, H.J. Kimble, Phys. Rev. Lett. 99, 180504 (2007).

[16] A. Al-Qasimi, D.F.V. James, Phys. Rev. A 77, 012117 (2008).

[17] E.T. Jaynes, F.W. Cummings, Proc. IEEE 51, 89 (1963).

[18] S. Bose, I. Fuentes-Guridi, P.L. Knight, V. Vedral, Phys. Rev. Lett. 87, 050401 (2001).

[19] M.S. Kim, Jinhyoung Lee, D. Ahn, P.L. Knight, Phys. Rev. A 65, 040101(R) (2002).

[20] S.B. Li, J.B. Xu, Phys. Lett. A 313, 175 (2003).

[21] J.H. Reinaa, A. Briridb, Microelectronics J. 39, 696 (2008).

[22] W.P. Li, Z.W. Wang, J.W. Yin, Y.F. Yu, J.L. Xiao, Physica B 403, 3709 (2008).

[23] D.L. Andrews, S. Naguleswaran, G.E. Stedman, Phys. Rev. A 57, 4925 (1998).

[24] A.D. Buckingham, P. Fischer, Phys. Rev. A 61, 035801 (2000).

[25] G.E. Stedman, S. Naguleswaran, D.L. Andrews, L.C. Dávila Romero, Phys. Rev. A 63, 047801 (2001).

[26] A.D. Buckingham, P. Fischer, Phys. Rev. A 63, 047802 (2001).

[27] D.L. Andrews, L.C. Dávila Romero, G.E. Stedman, Phys. Rev. A 67, 055801 (2003).

[28] P.W. Milonni, R.W. Boyd, Phys. Rev. A 69, 023814 (2004).

[29] P.R. Berman, R.W. Boyd, P.W. Milonni, Phys. Rev. A 74, 053816 (2006).

[30] P.W. Milonni, R. Loudon, P.R. Berman, S.M. Barnett, Phys. Rev. A 77, 043835 (2008).

[31] K. Huang, Statistical Mechanics, Wiley, New York 1963.

[32] D.N. Zubarev, Sov. Phys.-Usp. 3, 320 (1960).

[33] R. Loudon, S.M. Barnett, J. Phys. B, At. Mol. Opt. Phys. 39, S555 (2006).

[34] D.J. Masiello, G.C. Schatz, Phys. Rev. A 78, 042505 (2008).

[35] S. Mukamel, Phys. Rev. A 76, 021803(R) (2007).

[36] G. Baym, N.D. Mermin, J. Math. Phys. 2, 232 (1961).

[37] C.P. Slichter, Principles of Magnetic Resonance, Springer-Verlag, Berlin 1978.

[38] R. Hanson, B. Witkamp, L.M.K. Vandersypen, L.H. Willems van Beveren, J.M. Elzerman, L.P. Kouwenhoven, Phys. Rev. Lett. 91, 196802 (2003). 
[39] J.M. Elzerman, R. Hanson, L.H. Willems van Beveren, B. Witkamp, L.M.K. Vandersypen, L.P. Kouwenhoven, Nature 430, 431 (2004).

[40] F.G. Major, G. Werth, Phys. Rev. Lett. 30, 1155 (1973).

[41] U. Tanaka, H. Imajo, K. Hayasaka, R. Ohmukai, M. Watanabe, S. Urabe, Phys. Rev. A 53, 3982 (1996).

[42] L. Essen, E.G. Hope, D. Sutcliffe, Nature 189, 298 (1961).

[43] S. Penselin, T. Moran, V.W. Cohen, G. Winkler, Phys. Rev. 127, 524 (1962).

[44] D.L. Huber, J.H. Van Vleck, Rev. Mod. Phys. 38, 197 (1966).

[45] W. Götze, P. Wölfle, J. Low Temp. Phys. 5, 575 (1971).

[46] R. Pirc, B.G. Dick, Phys. Rev. B 10, 3594 (1974).
[47] W. Götze, G.M. Vujičić, Phys. Rev. B 38, 9398 (1988).

[48] R. Beck, W. Götze, P. Prelovšek, Phys. Rev. A 20, 1140 (1979).

[49] K.W. Becker, J. Keller, Z. Phys. B 62, 477 (1986).

[50] N. Bloembergen, E.M. Purcell, R.V. Pound, Phys. Rev. 73, 679 (1948).

[51] R. Kubo, in: Fluctuation, Relaxation and Resonance in Magnetic Systems, Ed. D. Ter Haar, Oliver and Boyd, Edinburgh 1962, p. 2368.

[52] M.I. Dyakonov, V.I. Perel, Sov. Phys.-JETP 33, 1053 (1971).

[53] D.W. Oxtoby, J. Chem. Phys. 70, 2605 (1979).

[54] J.H. Eberly, K. Wódkiewicz, B.W. Shore, Phys. Rev. A 30, 2381 (1984).

[55] A. Berthelot, I. Favero, G. Cassabois, C. Voisin, C. Delalande, P. Roussignol, R. Ferraira, J.M. Gérard, Nature Phys. 2, 759 (2006). 